\title{
Trace Element Analysis in Geochemical Systems by STEM/EDS
}

\author{
Z. Gainsforth ${ }^{1}$, K. Bustillo ${ }^{2}$, A. L. Butterworth ${ }^{1}$, R. C. Ogliore ${ }^{3}$, and A. J. Westphal ${ }^{1}$ \\ ${ }^{1}$ Space Sciences Laboratory, University of California Berkeley, 7 Gauss Way, Berkeley, CA, 94720 \\ 2. National Center for Electron Microscopy, Lawrence Berkeley Laboratory, MS 72-150, Berkeley, CA, \\ 94720. \\ 3. Hawai'i Institute of Geophysics and Planetology, University of Hawai 'i at Manoa, Honolulu, HI \\ 96822
}

Minor and trace elements have low concentrations (often $<1 \mathrm{wt} \%$ ) and usually do not greatly affect mineral structure. Therefore, they can have a simple relationship with temperature, fugacity, bulk composition, etc., and are very powerful geochemical indicators alongside major element compositions. However, measuring minor elements is often a challenge for many techniques.

Until recently, measurement of trace elements with ppm concentrations has been inaccessible to STEM EDS. Development of fast SDD detectors with solid angles $>1 / 2$ steradian and faster processors are now making such measurements a reality. STEM EDS measurements already have a low brehmsstrahlung background owing to thin samples. However, the combination of high beam currents required to attain sufficient $\mathrm{x}$-ray counts and high beam energy (typically 60-300 kV) tends to damage samples. The detection limits are therefore determined by the count rates obtainable from a given beam current, while still maintaining sample integrity.

Here we show trace element detections as low as $200 \mathrm{ppm}$ in geological and extraterrestrial samples. For many samples, trace element detection methods such as XRF, wet chemistry, etc., are not possible because of small sample size or mixed phases at the nanoscale. Therefore trace element detection by TEM becomes the best, and sometimes the only option. At ppm levels, pileup, spurious elements from the column and background subtraction become much more difficult to accommodate.

Our samples were embedded in epoxy, ultramicrotomed onto amorphous carbon TEM grids, and examined in an FEI Titan at 80-200 keV with a 0.7 steradian, 4 element SDD at the National Center for Electron Microscopy. All uncertainties are statistical $3 \sigma$ values determined from the goodness of the fit for the peak and background, not to be confused with accuracy.

A Fluorapatite standard (USNM 1044021, UCB 452) was previously characterized by wet chemistry, isotope dilution, and EPMA [1]. It contains 590 ppm Sr, 3400 ppm La, 4700 ppm Ce, 1100 ppm Nd, and $200 \mathrm{ppm}$ Th by weight. Using a $200 \mathrm{keV}$ beam at $0.7 \mathrm{nA}$, we mapped a region $4.5 \times 5.5 \mu \mathrm{m}$ in size for 28.5 hours. Quantification of rare earths agreed to within 10\% of the values determined by isotope dilution. Sr and Th similarly agreed within $20 \%$ of the standardized abundances. Na and P were lower than measured by EPMA likely from volatilization. This highlights the importance of understanding the volatilization sequence of a mineral before interpreting quantifications. In our case, we found that measurements of rare earths in apatites by TEM EDS should be robust. 
We measured trace Se in pyrrhotites from comet Wild 2 returned by NASA's Stardust mission [2]. These pyrrhotites would not be accessible to other techniques due to their small sample size and the spacecraft collection medium. Iron sulfides are known to be moderately tolerant of electron beam doses, but to monitor sample alteration, we obtained before and after HAADF images, and acquired at least one short (5 minute and/or 20 minute) EDS cube before the long acquisition. We saw no evidence of volatilization or mobility in any element including sulfur. Greshake [3] found that heated Orgueil pyrrhotites lost S and Se, with S volatilizing more rapidly than Se. Therefore the lack of volatilization of $\mathrm{S}$ means that Se did not volatilize in the TEM due to heating. Similarly, we claim that Se was not lost by sputtering since the lower mass of $\mathrm{S}$ would have caused it to sputter more rapidly than Se.

The terminal particle of track C2052,2,74 (Gigan) contains a pyrrhotite with $0.9 \pm 3 \% \mathrm{wt} \% \mathrm{Ni}$ and 200 $\pm 75 \%$ ppm of Se [3]. One of two terminal particles of track C2038,1,105 (Caligula) is a sulfide-silicate assemblage [4]. The largest sulfide contains $2700 \pm 75 \% \mathrm{ppm} \mathrm{Ni}, 280 \pm 25 \% \mathrm{ppm}$ Se, and $190 \pm 55 \%$ ppm $\mathrm{Rb}$. We have no information about volatilization of $\mathrm{Rb}$ in sulfides, and therefore $190 \mathrm{ppm}$ is a lower limit. The second terminal particle in track C2038,1,105 (Nadya) contains $1080 \pm 30 \%$ ppm Ni, $420 \pm 20 \%$ ppm Mn and $340 \pm 30 \%$ ppm Se. Finally, the terminal particle of track C2062,2,162 (Cecil) is a sulfide/silicate assemblage containing nanopyrrhotites [5]. One $300 \mathrm{~nm}$ wide pyrrhotite contains $400 \pm 30 \% \mathrm{ppm}$ Se and $3.6 \mathrm{wt} \% \mathrm{Ni}$.

For comparision, Greshake [3] measured Se in pyrrhotites from the Orgueil meteorite using XRF and found them to have concentrations between 36 and 100 ppm. Flynn [6] measured 100 ppm of Se in a pyrrhotite-dominated IDP.

The error bars on the above measurements are statistical only. Combined XRF and TEM/EDS using additional standards should allow the systematic errors to be reduced. We conclude that many trace elements in select phases can now be measured by STEM/EDS including rare earths in apatite and selenium in sulfides even within submicron grains. Reasonable counts on Se in nanosulfides allows for meaningful trace element geochemistry.

\section{References:}

[1] E. Jarosewich, et al., Geostandards Newsletter 1 (1980) 43-47.

[2] D. Brownlee et al., Science (2006) 314, 1711.

[3] A. Greshake, et al., Meteoritics and Planetary Science 33 (1998): 267-290. doi:10.1111/j.

1945-5100.1998.tb01632.x.

[3] R. C. Ogliore, et al., $43^{\text {rd }}$ Lunar and Planetary Sciences Conference (2012)

[4] Z. Gainsforth, et al., $44^{\text {th }}$ Lunar and Planetary Sciences Conference (2013)

[5] Z. Gainsforth, et al., $45^{\text {th }}$ Lunar and Planetary Sciences Conference (2014)

[6] G. J. Flynn, et al., Meteoritics and Planetary Science 35 (2000): 54.

[7] The operations of the National Center for Electron Microscopy at Lawrence Berkeley National Laboratory are supported by the Director, Office of Science, Office of Basic Energy Sciences, U.S. Department of Energy under contract number DE-AC02-05CH11231. 